\title{
A NODAL-BASED FINITE ELEMENT APPROXIMATION OF THE MAXWELL PROBLEM SUITABLE FOR SINGULAR SOLUTIONS
}

\author{
SANTIAGO BADIA* AND RAMON CODINA $^{\dagger}$
}

\begin{abstract}
A new mixed finite element approximation of Maxwell's problem is proposed, its main features being that it is based on a novel augmented formulation of the continuous problem and the introduction of a mesh dependent stabilizing term, which yields a very weak control on the divergence of the unknown. The method is shown to be stable and convergent in the natural $H(\operatorname{curl} ; \Omega)$ norm for this unknown. In particular, convergence also applies to singular solutions, for which classical nodal based interpolations are known to suffer from spurious convergence upon mesh refinement.
\end{abstract}

1. Introduction. The simulation of electromagnetic phenomena with increasing complexity demands accurate and efficient numerical methods suitable for large-scale computing. Finite element (FE) methods are commonly used in this context because they can easily handle complicated geometries by using unstructured grids, provide a rigorous mathematical framework and allow adaptation.

In many applications of current interest, the electromagnetic problem is coupled to other physical processes. Salient examples of multiphysics phenomena that include electromagnetics are magnetohydrodynamics (MHD) and plasma physics. These two problems have experienced increasing attention due to the need to develop numerical laboratories in fusion technology design. The simulation of these problems (and many others) would benefit of an all-purpose FE method that would be suitable for the different sub-problems at hand, simplifying the implementation issues and the enforcement of the coupling conditions. In particular, an all-purpose continuous nodal-based formulation would be a favored candidate. E.g. the Navier-Stokes equations are commonly solved with stabilized FE approximations that can deal with the singularly perturbed nature of the system for high Reynolds numbers and circumvent the restrictions related to the corresponding inf-sup condition (see e.g. $[13,14])$. In plasma physics, fields computed by discontinuous FE Maxwell solvers create a considerable numerical noise when embedded in a plasma code, e.g. using the particle-in-cell method (see [2]). Furthermore, nodal approximations are particularly well-suited for time-dependent electromagnetic problems because the mass matrix can be consistently lumped without loss of accuracy, leading to inexpensive transient solvers.

The Maxwell operator has a saddle-point structure, with the particularity that the Lagrange multiplier introduced to enforce the divergence-free constraint is identically zero. Existing FE methods that satisfy the discrete counterpart of the inherent inf-sup condition for this problem are based on Nedelec's or edge elements (see e.g. [27, 33]); edge elements lead to fields with discontinuous normal component on element edges or faces. We also refer to alternative formulations based on discontinuous Galerkin approximations [28, 24, 23, 34]. With the aim to solve the Maxwell problem with Lagrangian finite elements (FEs), the differential operator of the problem can be transformed into an elliptic one, by adding an exact penalty term containing the divergence (see [26]); the penalty is exact because the Lagrange multiplier vanishes. The resulting method satisfies the compatibility conditions over the element faces in a pointwise sense. Unfortunately, this method is not able to converge to nons-

*Centre Internacional de Metodes Numerics a l'Enginyeria (CIMNE), Parc Mediterrani de la Tecnologia, UPC, Esteve Terrades 5, 08860 Castelldefels, Spain (sbadia@cimne.upc.edu). The work of the first author was funded by the European Research Council under the FP7 Programme Ideas through the Starting Grant No. 258443 - COMFUS: Computational Methods for Fusion Technology.

†Universitat Politècnica de Catalunya, Jordi Girona 1-3, Edifici C1, 08034 Barcelona, Spain (ramon.codina@ upc.edu). 
mooth solutions that appear in nonconvex domains, e.g. domains with re-entrant corners (see $[25,17]$ and Section 3$)$.

Using an innovative idea, Costabel and Dauge proposed in [17] a rehabilitation of $H^{1}$ conforming $\mathcal{C}^{0}$ nodal (i.e. Lagrangian) FEs based on a weighted version of the penalty term that was able to converge to the "good" solution in nonconvex domains. In order to use the resulting numerical method, singularity regions have to be identified a priori, and proper weighted functions constructed, based on this information. In the negative side, it clearly complicates the numerical integration (of the weighted term), loses computational efficiency and complicates the automatization of the simulations. An alternative approach to solve the Maxwell problem is the decomposition of the solution into singular and smooth part (see $[2,26])$ but this method is harder to generalize, specially in three dimensions. Very recently, Duan et al. have designed in [19] a method based on local projections that uses a FE space composed of cubic nodal elements enriched with edge and element bubbles. The introduction of the local projection in the penalty term allows to converge to nonsmooth solutions, but the same projection weakens convergence, which is only attained in the $L^{2}$ norm. There are other nodal-based FE methods, but they converge to spurious solutions in nonconvex domains (see e.g. $[30,31])$.

In this work, we aim at developing a new mixed FE formulation for Lagrangian finite elements, based on a stabilized approximation of a novel augmented formulation of the Maxwell problem. We also refer to [6] for a similar approach, regarded to the eigenvalue problem. The compatibility condition associated to the inf-sup condition can be avoided by the introduction of the stabilization and exact penalty terms. The method can be understood as a residualbased FE method heuristically motivated in a variational multiscale framework [29]. On the other hand, the resulting numerical algorithm is able to capture nonsmooth solutions, so it is suitable for problems in nonconvex domains. The method is stable and convergent for any pair of nodal FE spaces for the unknown and the Lagrange multiplier. The implementation is straightforward, since the extra terms are standard and can be integrated numerically like the Galerkin terms. It can be implemented in a stabilized FE solver for the Navier-Stokes equations with minor modifications. Thus, the method is an excellent candidate for being used in MHD; we have developed a nodal-based FE formulation of the visco-resistive MHD problem where the magnetic sub-problem is approximated following the ideas in this work in [5], reporting excellent results.

The outline of the paper is as follows. In Section 2 we introduce the Maxwell problem and different augmented and/or penalized formulations. Section 3 is devoted to the numerical approximation of the problem by Lagrangian FEs. The problem related to nonconvex domains is discussed and the new formulation introduced. A complete stability and convergence analysis is also provided. In Section 4 we present some numerical experiments that confirm the theoretical analysis. Section 5 closes the article drawing some conclusions.

2. The Maxwell problem. In this section, we introduce some notation and state the Maxwell problem. We consider different augmented and penalized formulations that will be used throughout the paper.

2.1. Notation. Let $\Omega$ be a bounded domain in $\mathbb{R}^{d}$, with $d=2,3$ the space dimension. Given a Banach space $X$, we denote its associated norm by $\|\cdot\|_{X}$; for the sake of conciseness, we will omit the subscript for the $L^{2}(\Omega)$ space of square integrable functions. The space of vector-valued functions with components in $X$ is denoted by $X^{d}$. The dimension superscript will be omitted in the norm, i.e. we will simply denote its norm by $\|\cdot\|_{X}$ instead of $\|\cdot\|_{X^{d}}$. The dual space of $X$ is denoted as $X^{\prime}$. The inner product between two scalar or vector functions $f_{1}, f_{2} \in L^{2}(\Omega)$ is denoted by $\left(f_{1}, f_{2}\right)$, whereas $\left\langle f_{1}, f_{2}\right\rangle$ is used for a duality pairing. 
$W^{s, m}(\Omega)$ is used for the standard Sobolev space, with real coefficients $s \geq 0$ and $m \geq 1$. Hilbert spaces $W^{s, 2}(\Omega)$ are denoted by $H^{s}(\Omega)$. We write $H_{0}^{1}(\Omega)$ for the space of functions in $H^{1}(\Omega)$ with null trace on $\partial \Omega$. We will make use of the following spaces of vector fields:

$$
\begin{aligned}
& H(\operatorname{div} ; \Omega):=\left\{\mathbf{v} \in L^{2}(\Omega)^{d} \text { such that } \nabla \cdot \mathbf{v} \in L^{2}(\Omega)\right\}, \\
& H(\operatorname{curl} ; \Omega):=\left\{\mathbf{v} \in L^{2}(\Omega)^{d} \text { such that } \nabla \times \mathbf{v} \in L^{2}(\Omega)^{d}\right\},
\end{aligned}
$$

and the subspaces

$$
\begin{aligned}
& H(\operatorname{div} 0 ; \Omega):=\{\mathbf{v} \in H(\operatorname{div} ; \Omega) \text { such that } \nabla \cdot \mathbf{v}=0\}, \\
& H_{0}(\operatorname{curl} ; \Omega):=\{\mathbf{v} \in H(\operatorname{curl} ; \Omega) \text { such that } \mathbf{n} \times \mathbf{v}=\mathbf{0} \text { on } \partial \Omega\} .
\end{aligned}
$$

We use the notation $A \lesssim B$ to indicate that $A \leq C B$, where $A$ and $B$ are expressions depending on functions that in the discrete case may depend on the discretization as well, and $C$ is a positive constant.

2.2. Problem statement. In this work, we consider the Maxwell problem, which physically describes magnetostatics in a bounded domain $\Omega$ surrounded by a perfect conductor. Let us consider $\Omega \subset \mathbb{R}^{d}$ to be a simply connected nonconvex polyhedral domain with a connected Lipschitz continuous boundary $\partial \Omega$. Besides its range of applicability, this system of partial differential equations exhibits the mathematical complications encountered in more involved model problems (see e.g. [19, 10]). The Maxwell problem can be stated as a minimization problem that consists in finding the vectorial field $\mathbf{u}$ (magnetostatic field) that minimizes the potential

$$
\mathcal{E}(\mathbf{v})=\int_{\Omega}\left(\lambda|\nabla \times \mathbf{v}|^{2}-2 \mathbf{v} \cdot \mathbf{f}\right) \mathrm{d} \mathbf{x}
$$

with the constraint $\nabla \cdot \mathbf{v}=0$ and the homogeneous boundary condition $\mathbf{n} \times \mathbf{v}=\mathbf{0}$ over the boundary $\partial \Omega$, for some divergence-free datum $\mathbf{f} ; \lambda$ is a positive physical parameter.

2.3. Augmented and penalized formulations. The Maxwell problem can be recasted as a saddle-point problem by enforcing the divergence constraint with a Lagrange multiplier $p$. The Euler-Lagrange equations read as follows: seek a pair $(\mathbf{u}, p)$ solution of

$$
\begin{aligned}
\lambda \nabla \times(\nabla \times \mathbf{u})-\nabla p & =\mathbf{f}, \\
\nabla \cdot \mathbf{u} & =0,
\end{aligned}
$$

with $\mathbf{n} \times \mathbf{u}=\mathbf{0}$ and $p=0$ on $\partial \Omega$. As we will see later on, $p$ vanishes in the appropriate functional setting. Thus, the problem consists of finding $\mathbf{u}$ such that $\lambda \nabla \times \nabla \times \mathbf{u}=\mathbf{f}$ and $\nabla$. $\mathbf{u}=0$ on $\Omega$. It has motivated the exact penalty approach, in which the divergence constraint is penalized and the Lagrange multiplier eliminated; it consists of seeking $\mathbf{u}$ solution of

$$
\lambda \nabla \times \nabla \times \mathbf{u}-\lambda \nabla(\nabla \cdot \mathbf{u})=\mathbf{f} \quad \text { in } \Omega .
$$

The regularization requires to add the boundary condition $\nabla \cdot \mathbf{u}=0$ on the boundary $\partial \Omega$ (see [26]). This re-statement of the problem is (in principle) very appealing from a numerical point of view. However, as we will see in the next section, this exact penalty modifies the functional setting of the original problem, leading to spurious solutions for nonconvex domains.

The variational interpretation of the mixed problem (2.1) admits two functional settings. The so-called curl formulation reads as: find $\mathbf{u} \in H_{0}(\operatorname{curl} ; \Omega)$ and $p \in H_{0}^{1}(\Omega)$ such that

$$
\begin{aligned}
(\lambda \nabla \times \mathbf{u}, \nabla \times \mathbf{v})-(\nabla p, \mathbf{v}) & =(\mathbf{f}, \mathbf{v}), & & \forall \mathbf{v} \in H_{0}(\operatorname{curl} ; \Omega), \\
(\nabla q, \mathbf{u}) & =0, & & \forall q \in H_{0}^{1}(\Omega),
\end{aligned}
$$


where $\mathbf{f} \in H(\operatorname{div} 0 ; \Omega)$ is assumed. However, this is not the only functional setting in which the problem is well-posed; the $H^{1}(\Omega)$ regularity for $p$ can be "transferred" to $\mathbf{u}$, leading to a curl-div variational formulation: find $\mathbf{u} \in H_{0}(\operatorname{curl} ; \Omega) \cap H(\operatorname{div} ; \Omega)$ and $p \in L^{2}(\Omega) / \mathbb{R}$ such that

$$
\begin{aligned}
(\lambda \nabla \times \mathbf{u}, \nabla \times \mathbf{v})+(p, \nabla \cdot \mathbf{v}) & =(\mathbf{f}, \mathbf{v}), & & \forall \mathbf{v} \in H_{0}(\mathbf{c u r l} ; \Omega) \cap H(\operatorname{div} ; \Omega), \\
-(q, \nabla \cdot \mathbf{u}) & =0, & & \forall q \in L^{2}(\Omega) .
\end{aligned}
$$

On the other hand, the exact penalty method only allows a curl-div formulation. Thus, its variational form reads as: seek $\mathbf{u} \in H_{0}(\operatorname{curl} ; \Omega) \cap H(\operatorname{div} ; \Omega)$ such that

$$
(\lambda \nabla \times \mathbf{u}, \nabla \times \mathbf{v})+(\lambda \nabla \cdot \mathbf{u}, \nabla \cdot \mathbf{v})=(\mathbf{f}, \mathbf{v}),
$$

for any $\mathbf{v} \in H_{0}(\operatorname{curl} ; \Omega) \cap H(\operatorname{div} ; \Omega)$. For the sake of conciseness, we introduce the bilinear forms

$$
a(\mathbf{u}, \mathbf{v})=(\lambda \nabla \times \mathbf{u}, \nabla \times \mathbf{v}), \quad b(\mathbf{v}, p)=-(\nabla p, \mathbf{v}),
$$

and $c(\mathbf{u}, p ; \mathbf{v}, q)=a(\mathbf{u}, \mathbf{v})+b(\mathbf{v}, p)-b(\mathbf{u}, q)$. Let us also denote the Hilbert spaces $H_{0}(\operatorname{curl} ; \Omega)$ and $H_{0}^{1}(\Omega)$ by $V$ and $Q$ respectively, supplemented with the norms

$$
\begin{aligned}
& \|\mathbf{v}\|_{V}:=\|\mathbf{v}\|_{H(\operatorname{curl} ; \Omega)}=\frac{1}{\ell}\|\mathbf{v}\|+\|\nabla \times \mathbf{v}\|, \\
& \|q\|_{Q}:=\|q\|_{H_{0}^{1}(\Omega)}=\frac{1}{\ell}\|q\|+\|\nabla q\|,
\end{aligned}
$$

where $\ell=\ell(\Omega)$ is a constant with dimensions of length that makes the norms dimensionally consistent. In the following, $\ell$ will denote a length scale, not necessarily the same at different appearances. The norm associated to the product space $V \times Q$ is denoted by

$$
\|\mathbf{v}, q\|=\lambda^{\frac{1}{2}}\|\mathbf{v}\|_{V}+\ell \lambda^{-\frac{1}{2}}\|q\|_{Q}
$$

From the standard theory of saddle-point problems, well-posedness of the curl formulation (2.3) is proved in the next theorem.

THEOREM 2.1. The following inf-sup condition is satisfied,

$$
\inf _{(\mathbf{u}, p) \in V \times Q \backslash\{\mathbf{0}, 0\}} \sup _{(\mathbf{v}, q) \in V \times Q \backslash\{\mathbf{0}, 0\}} \frac{c(\mathbf{u}, p ; \mathbf{v}, q)}{\|\mathbf{u}, p\||\|\mid\| \mathbf{v}, q \|} \geq \beta>0 .
$$

As a consequence, formulation (2.3) is well-posed.

Proof. The form $a: V \times V \rightarrow \mathbb{R}$ is bilinear, continuous and coercive when it is restricted to $V \cap H(\operatorname{div} 0 ; \Omega)$ (the closed subspace of $V$ in the kernel of $b(\cdot, q)$ for any $q \in Q$ ), since $a(\mathbf{v}, \mathbf{v}) \geq \lambda\|\nabla \times \mathbf{v}\|^{2}$ for any $\mathbf{v} \in V \cap H(\operatorname{div} 0 ; \Omega)$. The $L^{2}(\Omega)$ control of $\mathbf{v}$ is consequence of the Poincaré-Friedrichs inequality

$$
\frac{1}{\ell}\|\mathbf{v}\| \leq c_{F}\|\nabla \times \mathbf{v}\|, \quad \forall \mathbf{v} \in V \cap H(\operatorname{div} 0 ; \Omega)
$$

(see [32, Corollary 3.51]). On the other hand, $b(\mathbf{v}, p)$ is a continuous bilinear form such that, for any $p \in Q$, there exists $\mathbf{v}_{p} \in V$ with $\left\|\mathbf{v}_{p}\right\|_{V}=1$ that satisfies $b\left(\mathbf{v}_{p}, p\right) \geq \beta_{b}\|p\|_{Q}$. This is true, since $\nabla p \in V$ for any $p \in Q$. The coercivity of $a$ in the kernel of $b$, and the inf-sup condition satisfied by $b$ are necessary and sufficient conditions for proving (2.8) 
(see [21, Proposition 2.36]). We know from the theory of saddle-point problems that (2.3) is well-posed if and only if condition (2.8) is satisfied (see [21, Theorem 2.34]).

The curl-div formulations are equivalent to the curl formulation (2.3).

Proposition 2.2. Formulations (2.4) and (2.5), with $\mathbf{f} \in H(\operatorname{div} 0 ; \Omega)$, are well-posed. Furthermore, they are equivalent to (2.3) in the sense that they lead to the same $\mathbf{u}$.

Proof. Let us only show that $p \equiv 0$ in (2.3), which will be systematically used along the paper. Taking $\mathbf{v}=\nabla p$ (which clearly belongs to $V$ ) in (2.3), and using the fact that $\nabla \times \nabla p=\mathbf{0}$ and $\nabla \cdot \mathbf{f}=0$ a.e. in $\Omega$, we obtain $\|\nabla p\|=0$. Since $p$ vanishes on $\partial \Omega$, it implies $p \equiv 0$ a.e. in $\Omega$ by virtue of Poincaré's inequality. We refer to [25, Propositions 3.4 and 3.5] for the completion of the proof. $\square$

2.4. A novel augmented formulation for the Maxwell problem. In this work, we propose a novel numerical approximation of the Maxwell problem whose starting point is a different augmented formulation. Since we are interested in a curl formulation for reasons that will become obvious in the next section, the idea consists of adding the term $\frac{\ell^{2}}{\lambda} \Delta p$ to (2.1b); $\ell>0$ is the penalty value, with dimension of length. A length scale is inherent to the problem, since it is needed to define dimensionally consistent norms in (2.6)-(2.7). Theoretically, this length scale comes from the Poincaré-Friedrichs inequality of the problem at hand. The augmented formulation in strong form consists of finding $\mathbf{u}$ and $p$ such that

$$
\begin{aligned}
\lambda \nabla \times \nabla \times \mathbf{u}-\nabla p & =\mathbf{f}, \\
-\nabla \cdot \mathbf{u}-\frac{\ell^{2}}{\lambda} \Delta p & =0,
\end{aligned}
$$

in $\Omega$, satisfying $\mathbf{n} \times \mathbf{u}=\mathbf{0}$ and $p=0$ on $\partial \Omega$. Since $p \in Q$ is identically zero, the penalty is exact. The weak form of the new formulation reads as: find $\mathbf{u} \in V$ and $p \in Q$ such that

$$
\begin{aligned}
a(\mathbf{u}, \mathbf{v})+b(\mathbf{v}, p) & =(\mathbf{f}, \mathbf{v}), & & \forall \mathbf{v} \in V, \\
-b(\mathbf{u}, q)+s_{p}(p, q) & =0, & & \forall q \in Q,
\end{aligned}
$$

where

$$
s_{p}(p, q)=\frac{\ell^{2}}{\lambda} \int_{\Omega} \nabla p \cdot \nabla q \mathrm{~d} \mathbf{x} .
$$

We now show the equivalence of the new formulation (2.9).

Proposition 2.3. Formulation (2.9) is well-posed and its solution $(\mathbf{u}, p)$ is the solution of (2.3) for $\mathbf{f} \in H(\operatorname{div} 0 ; \Omega)$.

Proof. Well-posedness is simply verified by proving that $p \equiv 0$ in (2.9) (using the ideas introduced above) and testing the system against $(\mathbf{v}, q)=(\mathbf{u}, p)$. The new formulation is clearly stable in the norm $\|\cdot\| \|$, because of the stability of the original curl formulation and the positivity of the term added. Equivalence is now straightforward.

\section{Numerical approximation.}

3.1. Finite element approximation. Let $\mathcal{T}_{h}$ be a partition of $\Omega$ into a set of finite elements $\{K\}$. For every element $K$, we denote by $h_{K}$ its diameter, and set the characteristic mesh size as $h=\max _{K \in \mathcal{T}_{h}} h_{K}$. We consider a non-degenerate family $\left\{\mathcal{T}_{h}\right\}_{h>0}$ of finite element partitions. The space of polynomials of degree less or equal to $k>0$ in a finite element $K$ is denoted by $\mathcal{P}_{k}(K)$. The space of continuous piecewise polynomials is defined as

$$
\mathcal{N}_{k}(\Omega)=\left\{v_{h} \in \mathcal{C}^{0}(\bar{\Omega}) \text { such that }\left.v_{h}\right|_{K} \in \mathcal{P}_{k}(K) \forall K \in \mathcal{T}_{h}\right\}
$$


This type of finite element space is the one that we consider in this work for both scalar fields and every component of vectorial fields. These approximations are usually called $H^{1}$ conforming approximations, because of the inter-element continuity. Any function $\mathcal{N}_{k}(\Omega)$ can be uniquely determined by its values on a set of points (nodes) in $\Omega$ (see [7, 21]), and so this is a nodal finite element approximation.

For quasi-uniform partitions, there is a constant $C_{\mathrm{inv}}$, independent of the mesh size $h$ (the maximum of all the element diameters), such that

$$
\left\|\nabla v_{h}\right\|_{L^{2}(K)} \leq C_{\mathrm{inv}} h_{K}^{-1}\left\|v_{h}\right\|_{L^{2}(K)}, \quad\left\|\Delta v_{h}\right\|_{L^{2}(K)} \leq C_{\mathrm{inv}} h_{K}^{-1}\left\|\nabla v_{h}\right\|_{L^{2}(K)}
$$

for all finite element functions $v_{h}$ defined on $K \in \mathcal{T}_{h}$. This inequality can be used for scalars, vectors or tensors.

3.2. The corner paradox. Although all the formulations introduced above are equivalent, stable and consistent, numerical approximations of the curl-div formulations (2.4) and (2.5) lead to spurious solutions for nonconvex domains, e.g. domains with re-entrant corners. Costabel provided in [15] a mathematical justification to this surprising observation.

LEMMA 3.1. If $\Omega$ is not convex, $V \cap H^{1}(\Omega)^{d}$ is a closed proper subspace of $V \cap$ $H(\operatorname{div} ; \Omega)$.

Out of this result, $H^{1}$-stable finite element formulations cannot converge to solutions in $V \cap H(\operatorname{div} ; \Omega)$ that do not belong to $V \cap H^{1}(\Omega)^{d}$. We can prove that this is the case of the curl-div formulation: find $\mathbf{u}_{h} \in X_{h} \subset H^{1}(\Omega)^{d} \cap V$ such that

$$
\left(\lambda \nabla \times \mathbf{u}_{h}, \nabla \times \mathbf{v}_{h}\right)+\left(\lambda \nabla \cdot \mathbf{u}_{h}, \nabla \cdot \mathbf{v}_{h}\right)=\left(\mathbf{f}, \mathbf{v}_{h}\right), \quad \forall \mathbf{v}_{h} \in X_{h}
$$

where $X_{h}$ is a $H^{1}$-conforming finite element space. From Lemma 3.1 we then have:

COROLLARY 3.2. If $\Omega$ is not convex

$$
\lim _{h \rightarrow 0}\left\|\mathbf{u}-\mathbf{u}_{h}\right\|_{V \cap H(\operatorname{div} ; \Omega)} \neq 0
$$

in general.

Proof. Every element of the sequence $\left\{\mathbf{u}_{h}\right\}_{h>0}$ belongs to $H^{1}(\Omega)^{d}$. Further, every $\mathbf{u}_{h}$ is solution of (3.3) and thus, $\lambda\left\|\nabla \times \mathbf{u}_{h}\right\|^{2}+\lambda\left\|\nabla \cdot \mathbf{u}_{h}\right\|^{2} \leq C\|\mathbf{f}\|\left\|\mathbf{u}_{h}\right\|$, for $C$ uniform with respect to $h$. From [15, Theorem 4.1], we have that $\left\|\mathbf{u}_{h}\right\|_{H^{1}(\Omega)} \lesssim\left\|\nabla \times \mathbf{u}_{h}\right\|+\left\|\nabla \cdot \mathbf{u}_{h}\right\|$, for $\Omega$ being a polyhedron (see also [15, Corollary 2.2] in the case when $\partial \Omega \in \mathcal{C}^{1,1}$ ). Thus, $\lambda\left\|\nabla \times \mathbf{u}_{h}\right\|+\lambda\left\|\nabla \cdot \mathbf{u}_{h}\right\| \lesssim\|\mathbf{f}\|$ and $\left\{\mathbf{u}_{h}\right\}_{h>0}$ is uniformly bounded in $H^{1}(\Omega)^{d} \subsetneq V \cap$ $H(\operatorname{div} ; \Omega)$ and cannot approximate an element in $V \cap H(\operatorname{div} ; \Omega)$ which is not in $H^{1}(\Omega)^{d}$.

This result implies that approximations based on (2.4) and (2.5) cannot capture solutions $\mathbf{u} \notin V \cap H^{1}(\Omega)^{d}$ of the Maxwell problem (2.3), and so, are not suitable for numerical purposes. This kind of solutions are called nonsmooth or singular solutions. Note that the key for this negative result is the spurious control on the divergence of the approximations based on (2.4) and (2.5), which implies that the whole gradient is uniformly bounded in $L^{2}(\Omega)$, since $\mathbf{u}_{h}$ is a $H^{1}(\Omega)^{d}$ function for all $h$.

Let us consider conforming finite element approximations of the spaces $V$ and $Q$, denoted by $V_{h}$ and $Q_{h}$ respectively. A crude Galerkin approximation of the curl conforming mixed problem (2.3) reads as: find $\mathbf{u}_{h} \in V_{h}$ and $p_{h} \in Q_{h}$ such that

$$
\begin{aligned}
a\left(\mathbf{u}_{h}, \mathbf{v}_{h}\right)+b\left(\mathbf{v}_{h}, p_{h}\right) & =\left(\mathbf{f}, \mathbf{v}_{h}\right), & & \forall \mathbf{v}_{h} \in V_{h}, \\
-b\left(\mathbf{u}_{h}, q_{h}\right) & =0, & & \forall q_{h} \in Q_{h} .
\end{aligned}
$$


The well-posedness of this finite dimensional problem relies on the discrete version of the inf-sup condition (2.8):

$$
\inf _{\left(\mathbf{u}_{h}, p_{h}\right) \in V_{h} \times Q_{h} \backslash\{\mathbf{0}, 0\}} \sup _{\left(\mathbf{v}_{h}, q_{h}\right) \in V_{h} \times Q_{h} \backslash\{\mathbf{0}, 0\}} \frac{c\left(\mathbf{u}_{h}, p_{h} ; \mathbf{v}_{h}, q_{h}\right)}{\left\|\mathbf{u}_{h}, p_{h}\right\| \mid\left\|\mathbf{v}_{h}, q_{h}\right\|} \geq \beta_{d}>0,
$$

for $\beta_{d}>0$ uniform with respect to $h$ (see e.g. [8]). As far as we know, it is not known whether there is any nodal interpolation for $V_{h} \times Q_{h}$ satisfying this inf-sup condition. However, it is satisfied when $V_{h}$ is given by the celebrated Nedelec's (or edge) elements; those elements are only conforming in $H$ (curl; $\Omega$ ), since they do not satisfy normal continuity over the element faces. A nodal finite element space can then be used for $Q_{h}$ (see e.g. [35]).

As a result, nodal finite elements have only been used with the "bad" formulation (3.3), leading to spurious solutions for nonconvex domains, e.g. domains with re-entrant corners. On the other hand, the "good" formulation (3.4) has been restricted to edge elements, since they do satisfy (3.5). Since the problem is the fact that a curl-div formulation is not suitable for numerical purposes, a rehabilitation of nodal finite elements has been proposed in [17]. The key idea of this approach is to introduce a weight in the penalty div-div term in (3.3) which depends on the distance to the singularities. The resulting problem is posed in a weighted Sobolev space that does satisfy an approximability property.

For the previous reasons, nodal elements have always been related to curl-div conforming formulations, whereas edge elements have always been related to curl formulations. Instead, in this article we construct a new curl mixed formulation and a corresponding residual-based stabilized finite element approximation that can be solved with nodal finite elements. Thus, our approach is very different to the one in [17]. Furthermore, the formulation we propose can be automatically used for any problem without the need to know where the singularities are and to define a weight function around every singularity.

3.3. A mixed finite element formulation suitable for nodal approximations. It is obvious that a nodal finite element approximation that would always provide the "physical" solution would be favored in many situations. In particular, the original motivation of this work lies in the multi-physics magnetohydrodynamics (MHD) problem. The numerical application of this phenomenon, with increasing interest in fusion reactor design, couples Navier-Stokes and Maxwell solvers. The ability to solve both problems with an all-purpose stabilized finite element method would make the extension of existing fluid solvers to MHD multi-physics very easy.

Our approach can be motivated as a residual-based stabilized discretization of the exact augmented formulation (2.9), although we will simply state the method without further heuristic motivation. The finite element formulation we propose is designed for $H^{1}$ conforming finite element spaces. Then, $V_{h}=\mathcal{N}_{k}(\Omega)^{d} \cap V$ and $Q_{h}=\mathcal{N}_{l}(\Omega) \cap Q$, for $k, l>0$ the order of approximation for $\mathbf{u}$ and $p$, respectively; there is no restriction between $k$ and $l$, and equal-order approximations are allowed. The method consists of seeking $\mathbf{u}_{h} \in V_{h}$ and $p_{h} \in Q_{h}$ solution of

$$
\begin{aligned}
a\left(\mathbf{u}_{h}, \mathbf{v}_{h}\right)+b\left(\mathbf{v}_{h}, p_{h}\right)+s_{u}\left(\mathbf{u}_{h}, \mathbf{v}_{h}\right) & =\left(\mathbf{f}, \mathbf{v}_{h}\right), & & \forall \mathbf{v}_{h} \in V_{h}, \\
-b\left(\mathbf{u}_{h}, q_{h}\right)+s_{p}\left(p_{h}, q_{h}\right) & =0, & & \forall q_{h} \in Q_{h},
\end{aligned}
$$

where the stabilization term reads

$$
s_{u}\left(\mathbf{u}_{h}, \mathbf{v}_{h}\right)=\sum_{K \in \mathcal{T}_{h}} c_{u} \lambda \int_{K} \frac{h_{K}^{2}}{\ell^{2}} \nabla \cdot \mathbf{u}_{h} \nabla \cdot \mathbf{v}_{h} \mathrm{~d} \mathbf{x},
$$


$c_{u}$ being an algorithmic constant. We can easily see that (3.6) is a residual-based FE approximation of the augmented formulation (2.9) (see e.g. [29, 12]). The stabilization parameter $c_{u} \lambda \frac{h_{K}^{2}}{\ell^{2}}$ must provide a dimensionally consistent method and it can be heuristically justified by using Fourier transform techniques (see e.g. [3]). The benefit of this approach is twofold: it allows us to circumvent the need of a discrete inf-sup condition and stabilizes singularly perturbed problems (see e.g. [21]).

The reason why the $s_{u}$ term is needed becomes evident from both theoretical analysis and numerical experimentation. Obviously, as $h \rightarrow 0$ this term vanishes, and the method is not a div-curl conforming algorithm. In the sequel, we analyze this method. We denote by

$$
c_{s}\left(\mathbf{u}_{h}, p_{h} ; \mathbf{v}_{h}, q_{h}\right)=c\left(\mathbf{u}_{h}, p_{h} ; \mathbf{v}_{h}, q_{h}\right)+s_{u}\left(\mathbf{u}_{h}, \mathbf{v}_{h}\right)+s_{p}\left(p_{h}, q_{h}\right)
$$

the stabilized counterpart of $c$.

3.3.1. Stability analysis. In the next theorem we establish stability of the bilinear form introduced above with respect to the mesh-dependent norm

$$
\left\|\mathbf{v}_{h}, q_{h}\right\|_{h}=\lambda^{\frac{1}{2}}\left\|\nabla \times \mathbf{v}_{h}\right\|+\lambda^{\frac{1}{2}}\left(\sum_{K \in \mathcal{T}_{h}} \frac{h_{K}^{2}}{\ell^{2}}\left\|\nabla \cdot \mathbf{v}_{h}\right\|_{K}^{2}\right)^{\frac{1}{2}}+\frac{\ell}{\lambda^{\frac{1}{2}}}\left\|\nabla q_{h}\right\| .
$$

LEMMA 3.3. The bilinear form $c_{s}: V_{h} \times Q_{h} \times V_{h} \times Q_{h} \rightarrow \mathbb{R}$ is coercive with respect to the mesh-dependent norm (3.8).

The proof of the lemma is straightforward. Unfortunately, this norm is not enough for numerical purposes, since it does not explicitly provide uniform control with respect to $h$ in $L^{2}(\Omega)$. However, we show in the next lemma that the "continuous" norm $\left\|\mathbf{u}_{h}, p_{h}\right\|$ for the FE solution can be bounded by its mesh-dependent norm.

LEMMA 3.4. The solution $\left(\mathbf{w}_{h}, \alpha_{h}\right) \in V_{h} \times Q_{h}$ of the discrete problem

$$
c_{s}\left(\mathbf{w}_{h}, \alpha_{h} ; \mathbf{v}_{h}, q_{h}\right)=\left\langle\mathbf{f}, \mathbf{v}_{h}\right\rangle+\left\langle g, q_{h}\right\rangle, \quad \forall\left(\mathbf{v}_{h}, q_{h}\right) \in V_{h} \times Q_{h}
$$

for $\mathbf{f} \in V^{\prime}$ and $g \in Q^{\prime}$, satisfies $\left\|\mathbf{w}_{h}, \alpha_{h}\right\|\|\| \mathbf{w}_{h}, \alpha_{h}\left\|_{h}+\right\| g \|_{Q^{\prime}}$. Furthermore, for any $\left(\mathbf{v}_{h}, q_{h}\right) \in V_{h} \times Q_{h}$, we have $\left\|\mathbf{v}_{h}, q_{h}\left|\left\|_{h} \lesssim\right\| \mathbf{v}_{h}, q_{h}\right|\right\|$.

Proof. Since $V_{h} \times Q_{h} \subset V \times Q$, by virtue of the continuous inf-sup condition (2.8), there exists $(\tilde{\mathbf{w}}, \tilde{\alpha}) \in V \times Q$ such that $\|\tilde{\mathbf{w}}, \tilde{\alpha}\|=1$ and

$$
c\left(\mathbf{w}_{h}, \alpha_{h} ; \tilde{\mathbf{w}}, \tilde{\alpha}\right) \geq \beta\left\|\mathbf{w}_{h}, \alpha_{h}\right\| \| .
$$

Let us denote by $\mathcal{S Z}_{h}(\cdot)$ the Scott-Zhang interpolation operator (see e.g. [7]) into the corresponding finite element space; the space (either $V_{h}$ or $Q_{h}$ ) is easily understood by the context. We have

$$
c\left(\mathbf{w}_{h}, \alpha_{h} ; \tilde{\mathbf{w}}, \tilde{\alpha}\right)=c\left(\mathbf{w}_{h}, \alpha_{h} ; \tilde{\mathbf{w}}, \tilde{\alpha}-\mathcal{S Z}_{h}(\tilde{\alpha})\right)+c\left(\mathbf{w}_{h}, \alpha_{h} ; \mathbf{0}, \mathcal{S} \mathcal{Z}_{h}(\tilde{\alpha})\right) .
$$

We bound the first term in the right-hand side as follows:

$$
\begin{aligned}
& c\left(\mathbf{w}_{h}, \alpha_{h} ; \tilde{\mathbf{w}}, \tilde{\alpha}-\mathcal{S Z}_{h}(\tilde{\alpha})\right) \\
& \quad \leq \lambda\left\|\nabla \times \mathbf{w}_{h}\right\|\|\nabla \times \tilde{\mathbf{w}}\|+\sum_{K \in \mathcal{T}_{h}}\left\|\nabla \cdot \mathbf{w}_{h}\right\|_{K}\left\|\tilde{\alpha}-\mathcal{S} \mathcal{Z}_{h}(\tilde{\alpha})\right\|_{K}+\left\|\nabla \alpha_{h}\right\|\|\tilde{\mathbf{w}}\| \\
& \quad \lesssim \lambda\left\|\nabla \times \mathbf{w}_{h}\right\|\|\nabla \times \tilde{\mathbf{w}}\|+\sum_{K \in \mathcal{T}_{h}} h_{K}\left\|\nabla \cdot \mathbf{w}_{h}\right\|_{K}\|\tilde{\alpha}\|_{H^{1}(\Omega)}+\left\|\nabla \alpha_{h}\right\|\|\tilde{\mathbf{w}}\| \\
& \quad \lesssim\left\|\mathbf{w}_{h}, \alpha_{h}\right\|\left\|{ }_{h}\right\| \tilde{\mathbf{w}}, \tilde{\alpha} \|,
\end{aligned}
$$


where we have used the interpolation properties of the Scott-Zhang projector (see e.g. [7]). Using the fact that $\left(\mathbf{w}_{h}, \alpha_{h}\right)$ is the solution of the stabilized problem (3.9), the second term in (3.10) can be treated as

$$
\begin{aligned}
c\left(\mathbf{w}_{h}, \alpha_{h} ; \mathbf{0}, \mathcal{S Z}_{h}(\tilde{\alpha})\right) & =\left\langle g, \mathcal{S Z}_{h}(\tilde{\alpha})\right\rangle-s_{p}\left(\alpha_{h}, \mathcal{S Z}_{h}(\tilde{\alpha})\right) \\
& \leq\|g\|_{Q^{\prime}}\left\|\mathcal{S} \mathcal{Z}_{h}(\tilde{\alpha})\right\|_{Q}+\frac{\ell^{2}}{\lambda}\left\|\nabla \alpha_{h}\right\|\left\|\nabla \mathcal{S} \mathcal{Z}_{h}(\tilde{\alpha})\right\| \\
& \leq\left(\left\|\mathbf{w}_{h}, \alpha_{h}\right\|_{h}+\|g\|_{Q^{\prime}}\right)\|\tilde{\mathbf{w}}, \tilde{\alpha}\|,
\end{aligned}
$$

by using the continuity of $\mathcal{S Z}_{h}(\cdot)$ in $H^{1}(\Omega)$. Since $\|\tilde{\mathbf{w}}, \tilde{\alpha}\|=1$ by construction, we get the upper bound for $\|\cdot\| \|$ in the lemma. The lower bound is easily obtained using an inverse inequality (see (3.2)).

REMARK 3.1. We infer from the previous lemma the importance of the $h\left\|\nabla \cdot \mathbf{w}_{h}\right\|$ stabilization term, which is essential for bounding $\left(\nabla \cdot \mathbf{w}_{h}, \tilde{\alpha}-\mathcal{S Z}_{h}(\tilde{\alpha})\right)$ in (3.11). In fact, the requirement of having this stabilization is not only technical, as is shown in Section 4 using numerical experimentation.

The following corollaries are consequences of Lemmata 3.3 and 3.4.

COROLlary 3.5. The stabilized bilinear form $c_{s}: V_{h} \times Q_{h} \times V_{h} \times Q_{h} \rightarrow \mathbb{R}$ is continuous with respect to the norm $\|\mid\| \|$.

Corollary 3.6. Problem (3.6) is well-posed, i.e. it admits a unique solution $\left(\mathbf{u}_{h}, p_{h}\right)$ bounded by the data as follows:

$$
\left\|\mathbf{u}_{h}, p_{h}\right\| \lesssim\|\mathbf{f}\|
$$

Proof. The coercivity in Lemma 3.3 with the upper bound in Lemma 3.4 for $g=0$ imply that

$$
\left\|\mathbf{u}_{h}, p_{h}\right\|^{2} \lesssim c_{s}\left(\mathbf{u}_{h}, p_{h} ; \mathbf{u}_{h}, p_{h}\right) .
$$

Therefore, (3.6) is a squared linear system of equations, with a positive definite system matrix. So, it proves existence and uniqueness of solutions. On the other hand, using the CauchySchwarz inequality we get $\left(\mathbf{f}, \mathbf{u}_{h}\right) \leq\|\mathbf{f}\|\left\|\mathbf{u}_{h}\right\| \leq\|\mathbf{f}\|\|\| \mathbf{u}_{h}, p_{h}\|\|$. Combining this result with (3.13), we prove the corollary. $\square$

Thus, the numerical approximation (3.6) is stable in the "continuous" norm. On the other hand, the consistency of the method is easily checked by the fact that both $p$ and $\nabla \cdot \mathbf{u}$ are zero a.e. in $\Omega$.

3.3.2. Error estimates. As commented above, numerical methods based on the curldiv formulation fail to converge to singular solutions due to the lack of an approximability condition (see Corollary 3.2). Formulation (3.6) avoids this problem, since both stability and continuity hold for the same norm $\|\cdot\| \|$ in which the continuous problem is well-posed.

In order to define the interpolation error function, we make use of the following result. We refer to [1, Proposition 3.7] for the proof of this lemma (see also [27, Lemma 4.2]).

LEMMA 3.7. If $\mathbf{v} \in V \cap H(\operatorname{div} ; \Omega)$ then $\mathbf{v} \in H^{r}(\Omega)^{d}$ for some real number $r>\frac{1}{2}$, and there holds

$$
\ell^{r-1}\|\mathbf{v}\|_{H^{r}(\Omega)} \lesssim\|\nabla \times \mathbf{v}\|+\|\nabla \cdot \mathbf{v}\|
$$

The previous lemma leads to the following result, that is used in the definition of the error interpolation function. 
Corollary 3.8. Any function $\mathbf{v} \in V \cap H(\operatorname{div} ; \Omega)$ belongs to $L^{2}(\partial K)$, for any $K \in$ $\mathcal{T}_{h}$.

Proof. As a consequence of the previous lemma, $\mathbf{v} \in H^{r}(K)^{d}$, for some $r>\frac{1}{2}$. Now, using the trace theorem for fractional Sobolev spaces in [18, Theorem 1], we obtain that $\mathbf{v} \in H^{r-\frac{1}{2}}(\partial K)$, which proves the result.

The interpolation error for the new formulation, which comes from the subsequent convergence analysis, is defined as

$$
E_{h}(\mathbf{u}, p):=\inf _{\left(\mathbf{w}_{h}, r_{h}\right) \in V_{h} \times Q_{h}} \varrho\left(\mathbf{u}-\mathbf{w}_{h}, p-r_{h}\right)
$$

where

$$
\varrho(\mathbf{v}, q):=\|\mathbf{v}, q\|+\lambda^{\frac{1}{2}}\left(\sum_{K} \frac{h_{K}}{\ell^{2}}\|\mathbf{v}\|_{L^{2}(\partial K)}^{2}\right)^{\frac{1}{2}} .
$$

THEOREM 3.9. The solution $\left(\mathbf{u}_{h}, p_{h}\right)$ of problem (3.6) for the family of finite element partitions $\left\{\mathcal{T}_{h}\right\}_{h>0}$ approximates the continuous solution $(\mathbf{u}, p)$ of problem $(2.3)$ in the following sense

$$
\left\|\mathbf{u}_{h}-\mathbf{u}, p_{h}-p\right\| \lesssim E_{h}(\mathbf{u}, p)
$$

Proof. On one hand, the Galerkin orthogonality, the consistency of the method and the fact that the finite element approximation is conforming lead to

$$
\begin{aligned}
& c_{s}\left(\mathbf{u}_{h}-\mathbf{w}_{h}, p_{h}-r_{h} ; \mathbf{v}_{h}, q_{h}\right)=c_{s}\left(\mathbf{u}-\mathbf{w}_{h}, p-r_{h} ; \mathbf{v}_{h}, q_{h}\right) \\
& \quad=c\left(\mathbf{u}-\mathbf{w}_{h}, p-r_{h} ; \mathbf{v}_{h}, q_{h}\right)+s_{u}\left(\mathbf{u}-\mathbf{w}_{h}, \mathbf{v}_{h}\right)+s_{p}\left(p-r_{h}, q_{h}\right)
\end{aligned}
$$

for any $\left(\mathbf{w}_{h}, r_{h}\right)$ and $\left(\mathbf{v}_{h}, q_{h}\right)$ in $V_{h} \times Q_{h}$. On the other hand, using integration by parts within each element domain $K \in \mathcal{T}_{h}$ for the $s_{u}$ term, we get:

$$
\begin{aligned}
s_{u}\left(\mathbf{u}-\mathbf{w}_{h}, \mathbf{v}_{h}\right)= & \sum_{K \in \mathcal{T}_{h}} c_{u} \lambda \int_{K} \frac{h_{K}^{2}}{\ell^{2}} \nabla \cdot\left(\mathbf{u}-\mathbf{w}_{h}\right) \nabla \cdot \mathbf{v}_{h} \mathrm{~d} \mathbf{x} \\
= & -\sum_{K \in \mathcal{T}_{h}} c_{u} \lambda \int_{K} \frac{h_{K}^{2}}{\ell^{2}}\left(\mathbf{u}-\mathbf{w}_{h}\right) \cdot \nabla \nabla \cdot \mathbf{v}_{h} \mathrm{~d} \mathbf{x} \\
& +\sum_{K \in \mathcal{T}_{h}} c_{u} \lambda \int_{\partial K} \frac{h_{K}^{2}}{\ell^{2}}\left(\mathbf{u}-\mathbf{w}_{h}\right) \cdot \mathbf{n} \nabla \cdot \mathbf{v}_{h} \mathrm{~d} \mathbf{x} \\
\lesssim & \sum_{K \in \mathcal{T}_{h}} c_{u} \lambda \frac{h_{K}^{2}}{\ell^{2}}\left\|\mathbf{u}-\mathbf{w}_{h}\right\|_{L^{2}(K)}\left\|\nabla \nabla \cdot \mathbf{v}_{h}\right\|_{L^{2}(K)} \\
& +\sum_{K \in \mathcal{T}_{h}} c_{u} \lambda \frac{h_{K}^{2}}{\ell^{2}}\left\|\mathbf{u}-\mathbf{w}_{h}\right\|_{L^{2}(\partial K)}\left\|\nabla \cdot \mathbf{v}_{h}\right\|_{L^{2}(\partial K)} .
\end{aligned}
$$

Using the inverse inequalities (3.2) and the relation $\left\|\phi_{h}\right\|_{L^{2}(\partial K)} \lesssim h_{K}^{-\frac{1}{2}}\left\|\phi_{h}\right\|_{L^{2}(K)}$, that holds for any piecewise polynomial function, together with Young's inequality and the conti- 
nuity of $c$ and $s_{p}$, we get

$$
\begin{aligned}
\frac{c_{s}\left(\mathbf{u}_{h}-\mathbf{w}_{h}, p_{h}-r_{h} ; \mathbf{v}_{h}, q_{h}\right)}{\left\|\mathbf{v}_{h}, q_{h}\right\| \|} \lesssim & \left\|\mathbf{u}-\mathbf{w}_{h}, p-r_{h}\right\| \\
& +\lambda^{\frac{1}{2}}\left(\sum_{K} \frac{h_{K}}{\ell^{2}}\left\|\mathbf{u}-\mathbf{w}_{h}\right\|_{L^{2}(\partial K)}^{2}\right)^{\frac{1}{2}} .
\end{aligned}
$$

By virtue of Lemma 3.4 with $(\mathbf{f}, g)=c_{s}\left(\mathbf{u}-\mathbf{w}_{h}, p-r_{h} ; \cdot, \cdot\right)$ and the fact that

$$
\|g\|_{Q^{\prime}}=\sup _{q \in Q \backslash\{0\}} \frac{-b\left(\mathbf{u}-\mathbf{w}_{h}, q\right)+s_{p}\left(p-r_{h}, q\right)}{\|q\|_{Q}} \lesssim\left\|\mathbf{u}-\mathbf{w}_{h}, p-r_{h}\right\|,
$$

we get:

$$
\begin{aligned}
\left\|\mathbf{u}_{h}-\mathbf{w}_{h}, p_{h}-r_{h}\right\| & \lesssim\left\|\mathbf{u}_{h}-\mathbf{w}_{h}, p_{h}-r_{h}\right\|_{h}+\left\|\mathbf{u}-\mathbf{w}_{h}, p-r_{h}\right\| \\
& \lesssim\left\|\mathbf{u}_{h}-\mathbf{w}_{h}, p_{h}-r_{h}\right\|_{h}+\varrho\left(\mathbf{u}-\mathbf{w}_{h}, p-r_{h}\right) .
\end{aligned}
$$

Testing (3.17) against $\left(\mathbf{v}_{h}, q_{h}\right)=\left(\mathbf{u}_{h}-\mathbf{w}_{h}, p_{h}-r_{h}\right)$ and using the coercivity of $c_{s}$ in Lemma 3.3, Cauchy-Schwarz and Young's inequalities, we obtain

$$
\begin{aligned}
\left\|\mathbf{u}_{h}-\mathbf{w}_{h}, p_{h}-r_{h}\right\|_{h}^{2} & \lesssim\left\|\mathbf{u}_{h}-\mathbf{w}_{h}, p_{h}-r_{h}\right\| \varrho\left(\mathbf{u}-\mathbf{w}_{h}, p-r_{h}\right) \\
& \lesssim\left(\left\|\mathbf{u}_{h}-\mathbf{w}_{h}, p_{h}-r_{h}\right\|_{h}+\varrho\left(\mathbf{u}-\mathbf{w}_{h}, p-r_{h}\right)\right) \varrho\left(\mathbf{u}-\mathbf{w}_{h}, p-r_{h}\right) \\
& \lesssim \frac{1}{4 \beta}\left\|\mathbf{u}_{h}-\mathbf{w}_{h}, p_{h}-r_{h}\right\|_{h}^{2}+(1+\beta) \varrho\left(\mathbf{u}-\mathbf{w}_{h}, p-r_{h}\right)^{2},
\end{aligned}
$$

for $\beta>0$. Taking $\beta$ large enough in (3.19) together with (3.18), we obtain $\| \mathbf{u}_{h}-\mathbf{w}_{h}, p_{h}-$ $r_{h} \| \lesssim \varrho\left(\mathbf{u}-\mathbf{w}_{h}, p-r_{h}\right)$. Combining this bound and the triangle inequality, we get:

$$
\left\|\mathbf{u}_{h}-\mathbf{u}, p_{h}-p\right\| \lesssim\left\|\mathbf{u}-\mathbf{w}_{h}, p-r_{h}\right\|+\left\|\mathbf{u}_{h}-\mathbf{w}_{h}, p_{h}-r_{h}\right\| \lesssim \varrho\left(\mathbf{u}-\mathbf{w}_{h}, p-r_{h}\right)
$$

for any $\left(\mathbf{w}_{h}, r_{h}\right) \in V_{h} \times Q_{h}$. Taking the infimum for $\mathbf{w}_{h} \in V_{h}$ and $r_{h} \in Q_{h}$, and invoking the expression for the interpolation error (3.14), we prove the theorem. $\square$

In the following, we obtain some a priori error estimates. Let us consider the interpolation estimates:

$$
\begin{aligned}
\inf _{\mathbf{w}_{h} \in V_{h}}\left\|\mathbf{v}-\mathbf{w}_{h}\right\|_{H^{s}(K)} \lesssim h_{K}^{t-s}\|\mathbf{v}\|_{H^{t}(K)}, & & 0 \leq s \leq t \leq k+1, \\
\inf _{r_{h} \in Q_{h}}\left\|q-r_{h}\right\|_{H^{s}(K)} \lesssim h_{K}^{t-s}\|q\|_{H^{t}(K)}, & & 0 \leq s \leq t \leq l+1,
\end{aligned}
$$

for any $K \in \mathcal{T}_{h}$ (see [17]). We get the following order of convergence for regular solutions, which in fact does not depend on the order $l$ of the approximation for $p$ :

COROLLARY 3.10. Let the solution of the continuous problem (2.3) be $\mathbf{u} \in H^{r}(\Omega)^{d}$, with $r \geq 1$. Then, the solution $\left(\mathbf{u}_{h}, p_{h}\right)$ of problem (3.6) satisfies the error estimate

$$
\left\|\mathbf{u}-\mathbf{u}_{h}, p-p_{h}\right\| \lesssim \lambda^{\frac{1}{2}} h^{t-1}\|\mathbf{u}\|_{H^{t}(\Omega)},
$$

where $t:=\min \{r, k+1\}$.

Proof. We infer from (3.21) that

$$
\inf _{\left(\mathbf{w}_{h}, r_{h}\right) \in V_{h} \times Q_{h}}\left\|\mathbf{u}-\mathbf{w}_{h}, p-r_{h}\right\| \lesssim \lambda^{\frac{1}{2}} h^{t-1}\|\mathbf{u}\|_{H^{t}(\Omega)},
$$


where we have used the fact that $p=0$ a.e. in $\Omega$. On the other hand, the trace inequality

$$
\|v\|_{L^{2}(\partial K)}^{2} \lesssim h_{K}^{-1}\|v\|_{L^{2}(K)}^{2}+h_{K}\|\nabla v\|_{L^{2}(K)}^{2}
$$

that holds for $v \in H^{1}(K), K \in \mathcal{T}_{h}$, allows us to obtain

$$
h_{K}\left\|\mathbf{u}-\mathbf{w}_{h}\right\|_{L^{2}(\partial K)}^{2} \lesssim\left\|\mathbf{u}-\mathbf{w}_{h}\right\|_{L^{2}(K)}^{2}+h_{K}^{2}\left\|\mathbf{u}-\mathbf{w}_{h}\right\|_{H^{1}(K)}^{2} .
$$

The proof follows by taking the infimum with respect to $\left(\mathbf{w}_{h}, r_{h}\right)$ in $(3.20)$, the previous result and (3.21). $\square$

We can prove a sharper a priori error estimate that is also applicable to nonsmooth solutions, under some assumptions over the partition $\mathcal{T}_{h}$ and/or the polynomial degree $k$ of $V_{h}$. In order to do that, we will make use of the following lemma and Lemma 3.7.

LEMmA 3.11. Let $\mathbf{u} \in V \cap H(\operatorname{div} ; \Omega)$ be the solution of (2.3). Then, $\mathbf{u}$ can be decomposed into a regular part and a singular part as follows:

$$
\mathbf{u}=\mathbf{u}_{0}+\nabla \varphi
$$

where $\mathbf{u}_{0} \in H^{1+r}(\Omega)^{d} \cap H_{0}(\mathbf{c u r l} ; \Omega), \varphi \in H_{0}^{1}(\Omega) \cap H^{1+r}(\Omega)$ for some real number $r>\frac{1}{2}$.

Lemma 3.11 is a consequence of the deep analysis about the singularities for the Maxwell problem due to Costabel and Dauge in [16] (see also [17, Section 6]).

Error estimates for nonsmooth solutions can be proved, relying on an assumption over the finite element space $V_{h}$ :

ASSUMPTION 3.1. There exists a finite element space $G_{h}$ defined over the mesh partition $\mathcal{T}_{h}$ such that, for any function $\phi_{h} \in G_{h}, \nabla \phi_{h} \in V_{h}$. Furthermore, this space satisfies the approximability property

$$
\inf _{\phi_{h} \in G_{h}}\left\|\phi-\phi_{h}\right\|_{H^{s}(K)} \lesssim h_{K}^{t-s}\|\phi\|_{H^{t}(K)}
$$

for any $K \in \mathcal{T}_{h}$, for $\phi \in H^{t}(K)$ and $0 \leq s \leq t \leq 1+k$.

Lemma 3.7 proves that the solution $\mathbf{u}$ of the Maxwell problem (2.3) for a forcing term $\mathbf{f} \in$ $H(\operatorname{div} 0 ; \Omega)$ belongs to $H^{r}(\Omega)^{d}$ for some $r>\frac{1}{2}$. Without any assumption over the regularity of the solution, we get the following error estimate that is based on the decomposition in Lemma 3.11:

COROLlARY 3.12. Under Assumption 3.1, the solution $\left(\mathbf{u}_{h}, p_{h}\right)$ of problem (3.6) satisfies the error estimate

$$
\left\|\mathbf{u}-\mathbf{u}_{h}, p-p_{h}\right\| \lesssim \sum_{K \in \mathcal{T}_{h}}\left(\lambda^{\frac{1}{2}} h_{K}^{t}\left\|\mathbf{u}_{0}\right\|_{H^{1+t}(K)}+\frac{\lambda^{\frac{1}{2}}}{\ell^{1-\epsilon}} h_{K}^{t-\epsilon}\|\varphi\|_{H^{1+t}(K)}\right),
$$

for any $\epsilon \in] 0, t-1 / 2[$ and for $t=\min \{r, k\}$.

Proof. Following [17], we use the decomposition $\mathbf{u}=\mathbf{u}_{0}+\nabla \varphi$ in Lemma 3.11 and consider optimal interpolations $\tilde{\mathbf{u}}_{0, h} \in V_{h}$ and $\tilde{\varphi}_{h} \in G_{h}$ for $\mathbf{u}_{0}$ and $\varphi$, respectively. Then, we have

$$
\begin{aligned}
\left\|\mathbf{u}_{0}-\tilde{\mathbf{u}}_{0, h}\right\|_{H^{s}(K)} & \lesssim h_{K}^{1+t-s}\left\|\mathbf{u}_{0}\right\|_{H^{1+t}(K)}, \\
\left\|\varphi-\tilde{\varphi}_{h}\right\|_{H^{s}(K)} & \lesssim h_{K}^{1+t-s}\|\varphi\|_{H^{1+t}(K)},
\end{aligned}
$$

for $0 \leq s \leq t+1$, with $t:=\min \{r, k\}$. These estimates also hold locally, within each element. Now, we pick $\mathbf{w}_{h}=\tilde{\mathbf{u}}_{0, h}+\nabla \tilde{\varphi}_{h} \in V_{h}$. We can easily see that

$$
\begin{aligned}
\left\|\mathbf{u}-\mathbf{w}_{h}, p\right\| \lesssim & \frac{\lambda^{\frac{1}{2}}}{\ell}\left\|\mathbf{u}_{0}-\tilde{\mathbf{u}}_{0, h}\right\|+\frac{\lambda^{\frac{1}{2}}}{\ell}\left\|\nabla\left(\varphi-\tilde{\varphi}_{h}\right)\right\| \\
& +\lambda^{\frac{1}{2}}\left\|\nabla \times\left(\mathbf{u}_{0}-\tilde{\mathbf{u}}_{0, h}\right)\right\|,
\end{aligned}
$$


where the contribution from $p$ has been neglected because $p=0$. For the second term in $E_{h}(\mathbf{u})$ we use

$$
h_{K}^{\frac{1}{2}}\left\|\mathbf{u}-\mathbf{w}_{h}\right\|_{L^{2}(\partial K)} \lesssim h_{K}^{\frac{1}{2}}\left\|\mathbf{u}_{0}-\tilde{\mathbf{u}}_{0, h}\right\|_{L^{2}(\partial K)}+h_{K}^{\frac{1}{2}}\left\|\nabla\left(\varphi-\tilde{\varphi}_{h}\right)\right\|_{L^{2}(\partial K)} .
$$

The first term in the right hand side of the previous inequality can be treated as above, using the trace inequality (3.23). For the second term, we use the embedding of $W^{\epsilon, m}(\partial K)$ into $W^{\epsilon+\frac{1}{m}, m}(K)$ (see [22]) for $\epsilon>0$ and $m=2$, getting:

$$
\begin{aligned}
h_{K}^{\frac{1}{2}}\left\|\nabla\left(\varphi-\tilde{\varphi}_{h}\right)\right\|_{L^{2}(\partial K)} & \lesssim h_{K}^{\frac{1}{2}} \ell^{\epsilon}\left\|\nabla\left(\varphi-\tilde{\varphi}_{h}\right)\right\|_{H^{\epsilon}(\partial K)} \\
& \lesssim h_{K}^{\frac{1}{2}} \ell^{\epsilon}\left\|\nabla\left(\varphi-\tilde{\varphi}_{h}\right)\right\|_{H^{\frac{1}{2}+\epsilon}(K)} \\
& \lesssim h_{K}^{\frac{1}{2}} \ell^{\epsilon}\left\|\varphi-\tilde{\varphi}_{h}\right\|_{H^{\frac{3}{2}+\epsilon}(K)} \\
& \lesssim h_{K}^{\frac{1}{2}} \ell^{\epsilon} h_{K}^{1+t-\frac{3}{2}-\epsilon}\|\varphi\|_{H^{1+t}(K)},
\end{aligned}
$$

where in the last step we have used the second interpolation estimate in (3.24) with $s=$ $\frac{3}{2}+\epsilon<1+t$. Note also that in the first step the fractional derivative in the norm in $H^{\epsilon}(\partial K)$ would scale as $h_{K}^{\epsilon}$, but we need to introduce a length scale $\ell$ independent of the element size to bound the whole $H^{\epsilon}(\partial K)$-norm.

Combining the previous results, we easily get the desired error estimate.

REMARK 3.2. When Assumption 3.1 is satisfied, the previous result is very strong, in the sense that we have not only proved convergence towards the good solution, but an (almost) optimal order of convergence, even for nonsmooth solutions. We can also weaken the approximability assumption over $G_{h}$, and in the limit case

$$
\lim _{h \rightarrow 0} \inf _{\phi_{h} \in G_{h}}\left\|\phi-\phi_{h}\right\|_{H^{s}(\Omega)}=0, \quad s \leq 1+r
$$

we would get strong convergence towards the solution without order. Alternatively, instead of considering the decomposition of $\mathbf{u}$, an interpolation result

$$
\lim _{h \rightarrow 0} \inf _{\mathbf{w}_{h} \in V_{h}}\left(\ell^{r-1}\left\|\mathbf{u}-\mathbf{w}_{h}\right\|_{H^{r}(\Omega)}+\left\|\nabla \times\left(\mathbf{u}-\mathbf{w}_{h}\right)\right\|\right)=0
$$

for $V_{h}$ would also lead to convergence towards the good solution, without the need to introduce $G_{h}$.

REMARK 3.3. Let us note that a similar method has recently been proposed in [6] for electromagnetic eigenvalue problems. The method in [6] depends on a coefficient $\alpha$ and corresponds to the method proposed herein for $\alpha=1$ with the only difference that no restriction over the FE spaces or meshes is assumed. Unfortunately, the convergence of the proposed algorithm is deteriorating in the limit $\alpha \rightarrow 1$ and the corresponding numerical analysis in [6] does not apply for the limit case considered in this work.

3.4. FE meshes and spaces satisfying Assumption 3.1. Assumption 3.1 is known to hold for $k \geq 4$ in dimension 2 without any assumption on the mesh typology. In this case, we can take $G_{h}$ as the finite element space obtained for the Argyris triangle. For $k \geq 2, G_{h}$ can be constructed by using the Bogner-Fox-Schmidt triangle; in order to do this, the triangulation $\mathcal{T}_{h}$ should admit a coarser mesh of macroelements. We refer to [17] for a detailed discusion.

For the most interesting case of linear interpolations, under the same kind of restriction over the mesh topology, the discrete space recently introduced in [36], based on a PowellSabin interpolant (see Figure 3.1 right), makes true Assumption 3.1 for $k \geq 1$, both in two 
and three dimensions (see also [9, 11]). Furthermore, we have observed from numerical experiments that a mesh with the crossed-box typology (see Figure 3.1 left) also satisfies this assumption. In a numerical code, it implies to perform a cheap pre-processing of the original mesh. Given any original triangular mesh, the Powell-Sabin mesh is obtained by introducing additional nodes on the mid-points of the edges and the element barycentes, and re-connecting the nodes properly. On the other hand, crossed-box meshes are obtained from a quadrilateral mesh by placing a node on its center, and creating four triangles; in fact, the additional node can be condensed. These are the two typologies of meshes considered in Section 4. We refer to [4] for detailed numerical experiments about the effect of having a suitable macro-element structure in the convergence of the method. In [5] we have extended this work to three-dimensions in the frame of MHD applications; we have considered both the $3 \mathrm{~d}$ Powell-Sabin element and a $3 \mathrm{~d}$ extension of the crossbox; both choices exhibit excellent convergence properties.
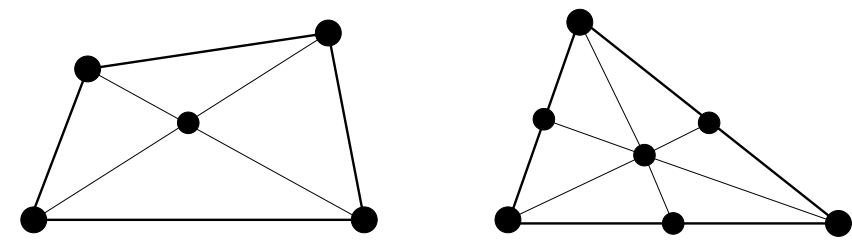

FIG. 3.1. Crossed-box (left) and Powell-Sabin (right) macro-element typologies.

\section{Numerical experiments.}

4.1. Stabilized curl formulation. In order to check, using numerical experimentation, that the nodal-based finite element approximation proposed in this article converges to both smooth and nonsmooth physical solutions, we take the datum $\mathbf{f}$ such that the solution of (2.3) in polar coordinates $(r, \theta)$ is:

$$
\mathbf{u}=\nabla\left(r^{\frac{2 n}{3}} \sin \frac{2 n \theta}{3}\right)
$$

in the nonconvex domain $\Omega \equiv[-1,1]^{2} \backslash[0,1]^{2}$, with one re-entrant corner. We have that $\mathbf{u} \in H^{\frac{2 n}{3}-\epsilon}(\Omega)^{2}$, for any $\epsilon>0$. Since for $n=1$ we have that $\mathbf{u} \notin H^{1}(\Omega)^{2}$, by virtue of Corollary 3.2, curl-div based finite element approximations converge to spurious solutions. On the other hand, as proved in Theorem 3.9, the solution of formulation (3.6) must converge to the physical solution (4.1) by using $h$-refinement and appropriate meshes. In order to observe this, we have considered a family of structured triangular meshes obtained by a partition of the domain into squares and a subsequent division of the squares in the crossed-box fashion (see Figure 3.1). We consider linear elements in the resulting mesh. The number of divisions in every direction has been set to $2^{i}$ with $i=3,4,5,6$; the characteristic mesh size $h$ is $2^{-i}$ and the number of triangular elements $2^{i+1}$. In Figure 4.1(a), we show the numerical errors $\mathbf{e}_{\mathbf{u}}=\mathbf{u}_{h}-\mathbf{u}$ and $\mathbf{e}_{p}=p_{h}-p$ for different norms as $h \rightarrow 0$. The convergence rate at every refinement level and numerical values of the error have been provided in Table 4.1. From these results, it is clear that the method we propose herein is capable to approximate numerically nonsmooth solutions, as Theorem 3.9 says. In fact, the order of convergence of the method is surprisingly high when compared to those for the weighted regularization in [15] and the discontinuous Galerkin technique in [28] (for the same test problem). Furthermore, optimal convergence in $L^{2}(\Omega)$ is obtained for this method. 


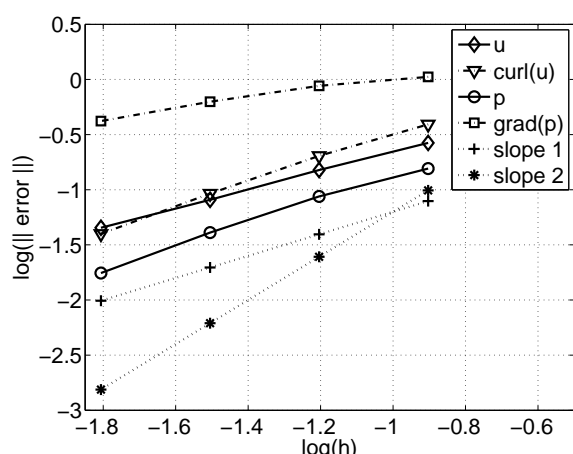

(a) $n=1$

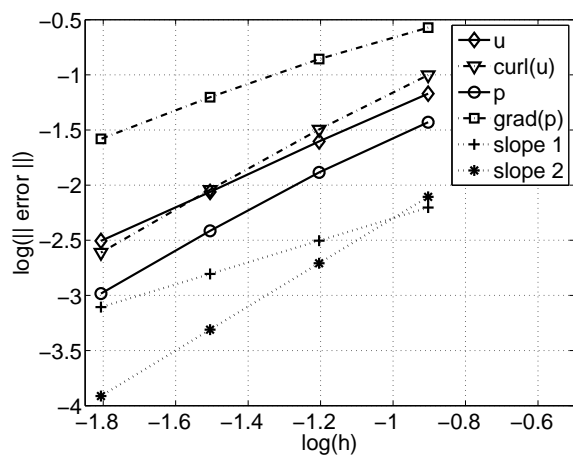

(c) $n=2$

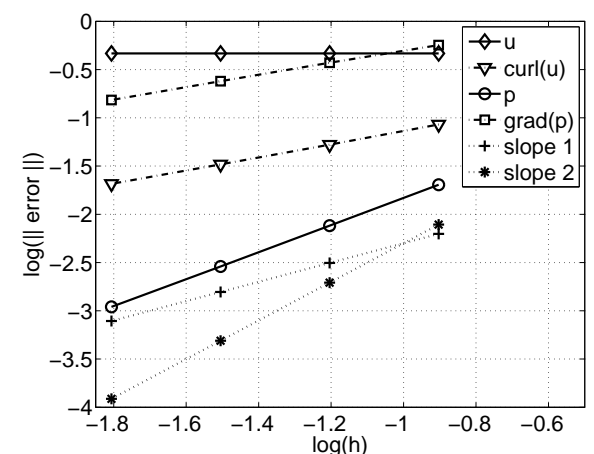

(b) $n=1$ without div-div term

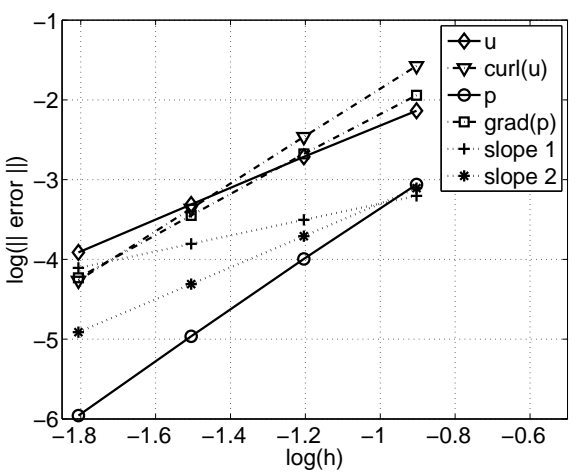

(d) $n=4$

FIG. 4.1. Error plots for different quantities in $L^{2}(\Omega)$ norm for Formulation (3.6) and the problem with analytical solution (4.1), with different values of $n$. Plot (b) corresponds to (3.6) without the stabilization term $s_{u}\left(\mathbf{u}_{h}, \mathbf{v}_{h}\right)$

TABLE 4.1

Experimental errors for Method (3.6) for $\mathbf{u}_{h}$ and rate of convergence (in brackets). Piecewise linear finite elements for both $\mathbf{u}_{h}$ and $p$.

\begin{tabular}{|c||c|c|c|c|c|c|}
\hline \multicolumn{1}{|c||}{} & \multicolumn{2}{|c|}{$n=1$} & \multicolumn{2}{c|}{$n=2$} & \multicolumn{2}{c|}{$n=4$} \\
\hline \hline & $\left\|\mathbf{e}_{\mathbf{u}}\right\|$ & $\left\|\nabla \times \mathbf{e}_{\mathbf{u}}\right\|$ & $\left\|\mathbf{e}_{\mathbf{u}}\right\|$ & $\left\|\nabla \times \mathbf{e}_{\mathbf{u}}\right\|$ & $\left\|\mathbf{e}_{\mathbf{u}}\right\|$ & $\left\|\nabla \times \mathbf{e}_{\mathbf{u}}\right\|$ \\
\hline \hline $2^{-3}$ & $2.67 \mathrm{e}-1(-)$ & $3.92 \mathrm{e}-1(-)$ & $6.75 \mathrm{e}-2(-)$ & $9.96 \mathrm{e}-2(-)$ & $7.31 \mathrm{e}-3(-)$ & $2.66 \mathrm{e}-2(-)$ \\
\hline $2^{-4}$ & $1.51 \mathrm{e}-1(0.82)$ & $2.03 \mathrm{e}-1(0.95)$ & $2.49 \mathrm{e}-2(1.44)$ & $3.20 \mathrm{e}-2(1.64)$ & $1.93 \mathrm{e}-3(1.92)$ & $3.44 \mathrm{e}-3(2.95)$ \\
\hline $2^{-5}$ & $8.11 \mathrm{e}-2(0.90)$ & $9.22 \mathrm{e}-2(1.14)$ & $8.68 \mathrm{e}-3(1.52)$ & $9.08 \mathrm{e}-3(1.82)$ & $4.89 \mathrm{e}-4(1.98)$ & $4.34 \mathrm{e}-4(2.99)$ \\
\hline $2^{-6}$ & $4.52 \mathrm{e}-2(0.84)$ & $3.98 \mathrm{e}-2(1.21)$ & $3.12 \mathrm{e}-3(1.48)$ & $2.44 \mathrm{e}-3(1.89)$ & $1.22 \mathrm{e}-4(2.00)$ & $5.43 \mathrm{e}-5(3.00)$ \\
\hline
\end{tabular}

Now, in order to stress the importance of the $h\left\|\nabla \cdot \mathbf{u}_{h}\right\|$ stabilization, we have switched off the term $\left(h_{K}^{2} \nabla \cdot \mathbf{u}_{h}, \nabla \cdot \mathbf{v}_{h}\right)$ from the formulation (3.6). In the previous stability analysis, this term is crucial for recovering $L^{2}(\Omega)$-control of $\mathbf{u}_{h}$. We perform the same convergence test as above and show the plots in Figure 4.1(b). As expected, convergence is not attained for the quantity $\left\|\mathbf{e}_{\mathbf{u}}\right\|$. So, the introduction of this term is motivated by both theoretical and numerical observations.

Going back to the full formulation (3.6), we perform the same convergence analysis with $n=2$ and $n=4$ in (4.1). In the case $n=2$, the solution $\mathbf{u}_{h}$ belongs to $H^{\frac{4}{3}-\epsilon}(\Omega)^{2} \subset$ 
TABLE 4.2

Experimental errors for Method (3.6) for $p_{h}$ and rate of convergence (in brackets). Piecewise linear finite elements for both $\mathbf{u}_{h}$ and $p$.

\begin{tabular}{|c||c|c|c|c|c|c|}
\hline \multicolumn{1}{|c||}{} & \multicolumn{2}{c|}{$n=1$} & \multicolumn{2}{c|}{$n=2$} & \multicolumn{2}{c|}{$n=4$} \\
\hline$h$ & $\left\|\mathbf{e}_{p}\right\|$ & $\left\|\nabla \mathbf{e}_{p}\right\|$ & $\left\|\mathbf{e}_{p}\right\|$ & $\left\|\nabla \mathbf{e}_{p}\right\|$ & $\left\|\mathbf{e}_{p}\right\|$ & $\left\|\nabla \mathbf{e}_{p}\right\|$ \\
\hline \hline $2^{-3}$ & $1.56 \mathrm{e}-1(-)$ & $1.05 \mathrm{e}+0(-)$ & $3.72 \mathrm{e}-2(-)$ & $2.68 \mathrm{e}-1(-)$ & $8.69 \mathrm{e}-4(-)$ & $1.14 \mathrm{e}-2(-)$ \\
\hline $2^{-4}$ & $8.70 \mathrm{e}-2(0.83)$ & $8.75 \mathrm{e}-1(0.27)$ & $1.30 \mathrm{e}-2(1.51)$ & $1.39 \mathrm{e}-1(0.95)$ & $1.01 \mathrm{e}-4(3.10)$ & $2.10 \mathrm{e}-3(2.44)$ \\
\hline $2^{-5}$ & $4.09 \mathrm{e}-2(1.09)$ & $6.29 \mathrm{e}-1(0.48)$ & $3.85 \mathrm{e}-3(1.76)$ & $6.27 \mathrm{e}-2(1.15)$ & $1.09 \mathrm{e}-5(3.22)$ & $3.56 \mathrm{e}-4(2.56)$ \\
\hline $2^{-6}$ & $1.76 \mathrm{e}-2(1.22)$ & $4.19 \mathrm{e}-1(0.59)$ & $1.04 \mathrm{e}-3(1.89)$ & $2.63 \mathrm{e}-2(1.25)$ & $1.10 \mathrm{e}-6(3.30)$ & $5.88 \mathrm{e}-5(2.60)$ \\
\hline
\end{tabular}

TABLE 4.3

Experimental errors for Method (3.6) for $\mathbf{u}_{h}$ and rate of convergence (in brackets) for the test problem with $n=1$ and Powell-Sabin triangle meshes. Piecewise linear finite elements for both $\mathbf{u}_{h}$ and $p$.

\begin{tabular}{|c||c|c|}
\hline \multicolumn{1}{|c||}{} & \multicolumn{2}{c|}{$n=1$} \\
\hline$h$ & $\left\|\mathbf{e}_{\mathbf{u}}\right\|$ & $\left\|\nabla \times \mathbf{e}_{\mathbf{u}}\right\|$ \\
\hline \hline $2^{-3}$ & $2.13 \mathrm{e}-1 \mathrm{e}-1(-)$ & $2.99 \mathrm{e}-1(-)$ \\
\hline $2^{-4}$ & $1.13 \mathrm{e}-1(0.91)$ & $1.40 \mathrm{e}-1(1.10)$ \\
\hline $2^{-5}$ & $5.98 \mathrm{e}-2(0.92)$ & $5.99 \mathrm{e}-2(1.22)$ \\
\hline $2^{-6}$ & $3.34 \mathrm{e}-2(0.84)$ & $2.48 \mathrm{e}-2(1.27)$ \\
\hline
\end{tabular}

$H^{1}(\Omega)^{2}$. Then, both curl-div and curl formulations are able to capture the solution. In any case, the smoothness of the solution does not allow us to obtain theoretically optimal convergence for first order approximation of both $\mathbf{u}_{h}$ and $p_{h}$, since $\mathbf{u} \notin H^{2}(\Omega)^{2}$. The convergence plot and convergence rates at every level of refinement can be found in Figure 4.1(c) and Table 4.2, respectively. The method exhibits some super-convergence. For $n=4$ the solution $\mathbf{u}$ belongs to $H^{\frac{8}{3}-\epsilon}(\Omega)^{2}$ and the optimal error estimate should apply. We can see that this is in fact the case for both $\mathbf{u}$ and $p$ in the continuous norm $\left\|\mathbf{e}_{\mathbf{u}}, \mathbf{e}_{p}\right\|$ in Figure 4.1(d) and Table 4.1. Again, the method exhibits super-convergence.

Finally, we solve the singular problem (with $n=1$ ) with a Powell-Sabin mesh. As expected, the method shows a very similar convergence order as the one obtained for crossedbox meshes. The numerical errors and slopes with respect to $h$ are shown in table 4.3.

Let us remark the fact that the stabilized finite element formulation (3.6) leads to a positive-definite linear system. In this work, this linear system has been solved using a direct solver. For larger scale problems, a Krylov iterative solver with a Schur complement type preconditioner could be explored (see [20]). This type of block-preconditioner allows one to decouple the computation of $\mathbf{u}_{h}$ and $p_{h}$ at the preconditioner level, reducing the original problem into two smaller ones, for which effective preconditioners can be used.

4.2. Stabilized curl-div formulation. Following the same idea as at the continuous level, in which we went from (2.3) to (2.4) passing regularity from $p$ to $\mathbf{u}$, we can pass from (3.6) to a curl-div stabilized finite element formulation. Proceeding this way, we get the discrete problem: find $\mathbf{u}_{h} \in V_{h}$ and $p_{h} \in Q_{h}$ solution of

$$
\begin{array}{rlrl}
a\left(\mathbf{u}_{h}, \mathbf{v}_{h}\right)+b\left(\mathbf{v}_{h}, p_{h}\right)+\left(c_{u} \lambda \nabla \cdot \mathbf{u}_{h}, \nabla \cdot \mathbf{v}_{h}\right) & =\left(\mathbf{f}, \mathbf{v}_{h}\right), & & \forall \mathbf{v}_{h} \in V_{h}, \\
-b\left(\mathbf{u}_{h}, q_{h}\right)+\sum_{K \in \mathcal{T}_{h}} \int_{K} \frac{h_{K}^{2}}{\lambda} \nabla p_{h} \cdot \nabla q_{h} \mathrm{~d} \mathbf{x}=0, & \forall q_{h} \in Q_{h} .
\end{array}
$$

Again, this method is a residual-based finite element method, in which the stabilization parameter has been chosen to be $c_{u} \lambda$. The second term in the right-hand side comes from the penalty term in (2.9) but taking $\frac{h_{K}^{2}}{\lambda}$ as penalty coefficient. The numerical analysis of this 
method uses similar arguments to the ones for (3.6). Since we have control over both the curl and the divergence of $\mathbf{u}_{h}$, and the control $h\left\|\nabla p_{h}\right\|$ only leads to $L^{2}(\Omega)$ stability for $p_{h}$, this problem is well-posed for the curl-div norm, for which there is no approximability property. Thus, this formulation is not able to deal with the singular solution (4.1) with $n=1$; we show this in Figure 4.2(a). However, as expected, the method converges for $n=2$ and $n=4$ to the good solution, since $\mathbf{u} \in H^{1}(\Omega)$. We show the error plots in Figures 4.2(b) and 4.2(c). Let us point out that in the curl-div formulation there is no control over $\nabla p_{h}$, and so, no convergence can be expected for it (see Figure 4.2(b)).

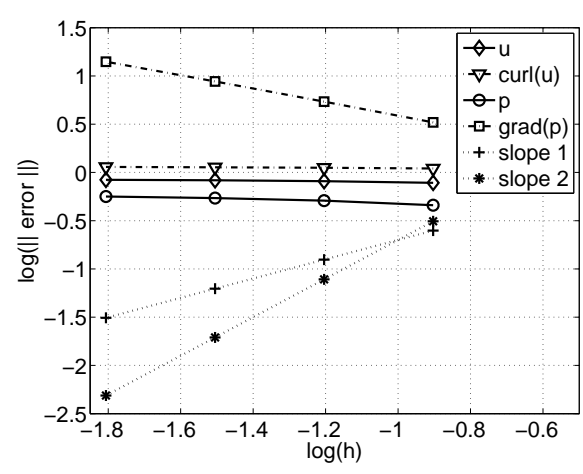

(a) $n=1$

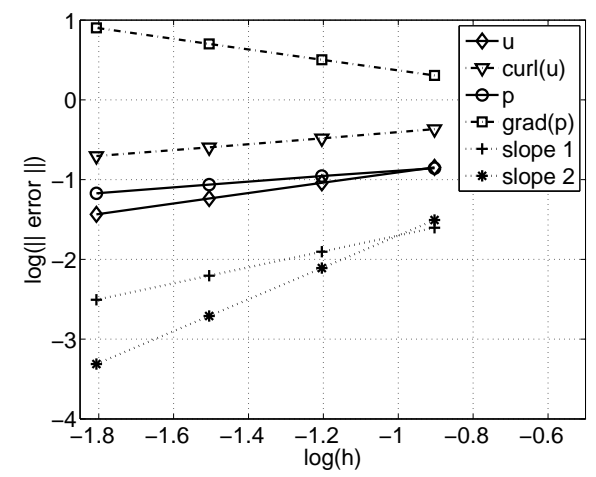

(b) $n=2$

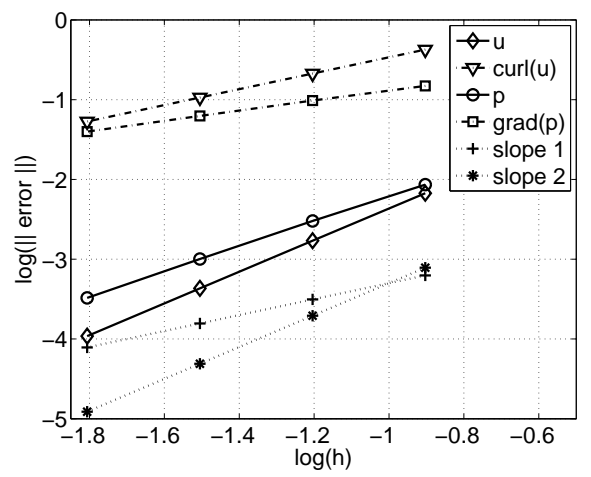

(c) $n=4$

FIG. 4.2. Error plots for different quantities in $L^{2}(\Omega)$ norm for Formulation (4.2) and the problem with analytical solution (4.1), with different values of $n$.

5. Conclusions. The finite element formulation proposed in this paper to approximate Maxwell's problem has been shown to allow one to use continuous Lagrangian interpolations for the unknown, yielding stable and convergent approximations to any solution of the continuous problem, including singular solutions. Convergence to smooth solutions is reached with optimal order.

The essential point to converge to singular solutions is to avoid the spurious control on the $L^{2}(\Omega)$-norm of the divergence of the unknown, typical of penalized or curl-div formulations. Instead of avoiding this by using weighted $L^{2}(\Omega)$-inner products, we resort to the introduction of a Lagrange multiplier to enforce the zero divergence restriction. However, to ensure stability of this in the appropriate functional setting, a novel augmented formula- 
tion has been introduced, which consists of adding a Laplacian of the multiplier in the zero divergence restriction. Since the multiplier is zero in the continuous problem, consistency remains unaltered. The final ingredient is to use a stabilized formulation at the discrete level, in our case consisting only in adding a least-square form of the zero divergence condition. The stabilizing term is multiplied by the square of the mesh size, so that it mimics stability of the divergence of the unknown in $H^{-1}(\Omega)$, not in $L^{2}(\Omega)$, as curl-div formulations wrongly do. This new term is also responsible for obtaining stability in the $L^{2}(\Omega)$ part of the whole $H(\operatorname{curl} ; \Omega)$ norm of the unknown. Finally, in order to have approximability for linear Lagrangian elements, particular mesh typologies must be used for singular solutions that can easily be generated by a cheap post-processing of any original triangular or quadrilateral mesh, both in two and three dimensions.

A classical numerical test has been used to check the theoretical predictions. Notably, very good convergence has been observed in the case when the solution is singular, as compared to other formulations that can be found in the literature.

The practical interest of our approach is clear. Even if tailored approximations for Maxwell's problem may be afforded at a reasonable computational cost when it is an isolated problem, it is obvious that a classical Lagrangian type approximation greatly simplifies its implementation in situations where this problem is coupled to others, as in MHD (see [5]). On the other hand, our approach may be viewed as an alternative to the use of the so called compatible discretization, satisfying the appropriate inf-sup conditions. In simple model problems, such as Stokes', Maxwell's and Darcy's, our formulation allows us to use the same interpolation for the unknowns in all cases, instead of one compatible for each case.

\section{REFERENCES}

[1] C. Amrouche, C. Bernardi, M. Dauge, and V. Girault. Vector potentials in three-dimensional non-smooth domains. Mathematical Methods in the Applied Sciences, 21(9):823-864, 1998.

[2] F. Assous, P. Ciarlet Jr., S. Labrunie, and J. Segré. Numerical solution to the time-dependent Maxwell equations in axisymmetric singular domains: the singular complement method. Journal of Computational Physics, 191:147-176, 2003.

[3] S. Badia and R. Codina. Unified stabilized finite element formulations for the Stokes and the Darcy problems. SIAM Journal on Numerical Analysis, 47(3):1977-2000, 2009.

[4] S. Badia and R. Codina. A combined nodal continuous-discontinuous finite element formulation for the Maxwell problem. Applied Mathematics and Computation, in press.

[5] S. Badia, R. Codina, and R. Planas. On an unconditionally convergent stabilized finite element approximation of resistive magnetohydrodynamics. Submitted.

[6] A. Bonito and J. Guermond. Approximation of the eigenvalue problem for the time harmonic Maxwell system by continuous Lagrange finite elements. Mathematics of Computation, in press, 2011.

[7] S.C. Brenner and L.R. Scott. The Mathematical Theory of Finite Element Methods. Springer-Verlag, 1994.

[8] F. Brezzi and M. Fortin. Mixed and Hybrid Finite Element Methods. Springer Verlag, 1991.

[9] A. Buffa, P. Ciarlet Jr., and E. Jamelot. Solving electromagnetic eigenvalue problems in polyhedral domains with nodal finite elements. Numerische Mathematik, 113(4):497-518, 2009.

[10] P. Ciarlet Jr. Augmented formulations for solving Maxwell equations. Computer Methods in Applied Mechanics and Engineering, 194(559-586), 2005.

[11] P. Ciarlet Jr. and G. Hechme. Mixed, augmented variational formulations for Maxwell's equations: numerical analysis via the macroelement technique. Submitted, 2009.

[12] R. Codina. A stabilized finite element method for generalized stationary incompressible flows. Computer Methods in Applied Mechanics and Engineering, 190:2681-2706, 2001.

[13] R. Codina. Analysis of a stabilized finite element approximation of the Oseen equations using orthogonal subscales. Applied Numerical Mathematics, 58:264-283, 2008.

[14] R. Codina, J. Principe, O. Guasch, and S. Badia. Time dependent subscales in the stabilized finite element approximation of incompressible flow problems. Computer Methods in Applied Mechanics and Engineering, 196:2413-2430, 2007

[15] M. Costabel. A coercive bilinear form for Maxwell's equations. Journal of Mathematical Analysis and Applications, 157(2):527-541, 1991. 
[16] M. Costabel and M. Dauge. Singularities of electromagnetic fields in polyhedral domains. Archives for Rational Mechanics and Analysis, 151(3):221-276, 2000.

[17] M. Costabel and M. Dauge. Weighted regularization of Maxwell equations in polyhedral domains. Numerische Mathematik, 93(2):239-277, 2002.

[18] Z. Ding. A proof of the trace theorem of Sobolev spaces on Lipschitz domains. Proceedings of the American Mathematical Society, 124(2):591-600, 1996.

[19] H.Y. Duan, F. Jia, P. Lin, and R.C.E. Tan. The local $L^{2}$ projected $C^{0}$ finite element method for Maxwell problem. SIAM Journal on Numerical Analysis, 47(2):1274-1303, 2009.

[20] H. Elman, D. Silvester, and A. Wathen. Finite Elements and Fast Iterative Solvers. Oxford Science Publications, 2005

[21] A. Ern and J.L. Guermond. Theory and Practice of Finite Elements. Springer-Verlag, 2004.

[22] V. Girault and P.A. Raviart. Finite Element Methods for Navier-Stokes Equations. Springer-Verlag, 1986.

[23] M. Grote, A. Schneebeli, and D. Schötzau. Interior penalty discontinuous Galerkin method for Maxwell's equations: energy norm error estimates. Journal of Computational and Applied Mathematics, 204(375386), 2007.

[24] M.J. Grote, A. Schneebeli, and D. Schötzau. Interior penalty discontinuous Galerkin method for Maxwell's equations: optimal $L^{2}$-norm error estimates. IMA Journal of Numerical Analysis, 28:440-468, 2008.

[25] C. Hazard. Numerical simulation of corner singularities: a paradox in Maxwell-like problems. Comptes Rendus-Mécanique, 330(1):57-68, 2002.

[26] C. Hazard and M. Lenoir. On the solution of time-harmonic scattering problems for Maxwell's equations. SIAM Journal on Mathematical Analysis, 6(1597-1630):1996, 27.

[27] R. Hiptmair. Finite elements in computational electromagnetism. Acta Numerica, 11(237-339), 2003.

[28] P. Houston, I. Perugia, and D. Schötzau. Mixed discontinuous Galerkin approximation of the Maxwell operator. SIAM Journal on Numerical Analysis, 42(1):434-459, 2005.

[29] T.J.R. Hughes. Multiscale phenomena: Green's function, the Dirichlet-to-Neumann formulation, subgrid scale models, bubbles and the origins of stabilized formulations. Computer Methods in Applied Mechanics and Engineering, 127:387-401, 1995.

[30] B. Jiang, J. Wu, and L.A. Povinelli. The origin of spurious solutions in computational electromagnetics. Journal of Computational Physics, 125(1):104-123, 1996.

[31] J. Jin. The Finite Element Method in Electromagnetics. Wiley, 1993.

[32] P. Monk. Finite Element Methods for Maxwell's Equations. Oxford University Press, 2003.

[33] S. Nicaise. Edge elements on anisotropic meshes and approximation of the Maxwell equations. SIAM Journal on Numerical Analysis, 39(3):784-816, 2001.

[34] I. Perugia, D. Schötzau, and P. Monk. Stabilized interior penalty methods for the time-harmonic Maxwell equations. Computer Methods in Applied Mechanics and Engineering, 191:4675-4697, 2002.

[35] D. Schötzau. Mixed finite element methods for stationary incompressible magneto-hydrodynamics. Numerische Mathematik, 96:771-800, 2004.

[36] T. Sorokina and A.J. Worsey. A multivariate Powell-Sabin interpolant. Advances in Computational Mathematics, 29(1):71-89, 2008. 